\author{
Richarr. J. Cyr \\ Pennsylvania State University \\ 208 Mueller Lab \\ University Park, PA \\ 16802 \\ Voice Phone\#- 1-814-865-6416 \\ FAX Phone\#- 1-814-865-9131 \\ Internet-RJC8@PSUVM.PSU.EDU
}




\title{
Calmodulin Immunolocalization to Cortical Microtubules is Calcium Independent
}

\author{
Deborah D. Fisher and Richard J. Cyr \\ Department of Biology \\ Pennsylvania State University \\ 208 Mueller Lab \\ University Park, PA
}

16802

\section{Manuscript received \\ Manuscript accepted \\ DISCLAIMER}

\begin{abstract}
This report was prepared as an account of work sponsored by an agency of the United Staies Government. Neither the United States Government nor any agency thereof, nor any of their employees, makes any warranty, express or implied, or assumes any legal liability or responsibility for the accuracy, completeness, or usefulness of any information, apparatus, product, or process disclosed, or represents that its use would not infringe privately owned rights. Reference herein to any specific commercial product, process, or service by trade name, trademark, manufacturer, or otherwise does not necessarily constitute or imply its endorsement, recommendation, or favoring by the United States Government or any agency thereof. The views and opinions of authors expressed herein do not necessarily state or reflect those of the United States Government or any agency thereof.
\end{abstract}


Supported by grants from the U.S. Department of Energy, U.S. Department of Agriculture, and the Pennsylvania state UniversityIntercollege Research Program

Corresponding Author: Richard J. Cyr, 208 Mueller Lab, Pennsylvania State University, University Park, PA 16802

Abbreviations: APM, amiprophos-methyl; CaM, calmodulin; FITC, fluorescein isothiocyanate; HAT, hypoxanthine/aminopterin/thymidine; mAb, monoclonal antibody; MT, microtubule; PM, Pipes and magnesium buffer; PVDF, polyvinylidene fluoride; $\mathbf{W} 7, \mathrm{~N}-(6$-aminohexyl)-5-chloro-1napthalenesulfonamide 
ABSTRACT

Calcium affects the stability of cortical microtubules (MTs) in lysed protoplasts. This calmodulin (CaM)-mediated interaction may provide a mechanism that serves to integrate cellular behavior with MT function. To test the hypothesis that CaM associates with these MTs, monoclonal antibodies were produced against CaM, and one (designated mAb1D10), was selected for its suitability as an immunocytochemical reagent. It is shown that CaM associates with the cortical MTs of cultured carrot (Daucus carota L.) and tobacco (Nicotiana tobacum L.) cells. Inasmuch as CaM interacts with calcium and affects the behavior of these MTs, we hypothesized that calcium would alter this association. To test this, protoplasts containing taxol-stabilized MTs were lysed in the presence of various concentrations of calcium and examined for the association of CaM with cortical MTs. At $1 \mu \mathrm{M}$ calcium, many protoplasts did not have CaM in association with the cortical MTs, while at $3.6 \mu \mathrm{M}$ calcium, this association was completely abolished. Control experiments were performed to eliminate alternate explanations including; differential antibody binding in the presence of calcium, detergent-induced redistribution of antigen, and epitope masking. The results are discussed in terms of a model in which CaM associates with MTs via two types of interactions; one calcium dependent and one independent. 


\section{INTRODUCTION}

Higher plant intracellular calcium concentrations fluctuate in response to a number of stimuli and numerous correlative and experimental studies have established this ion as an important second messenger (Marmé, 1988; Gilroy et al., 1990). However, it remains unclear how changes in calcium levels affect relevant targets. One model for the second messenger role of calcium suggests that this ion interacts with calcium binding proteins, forming complexes that subsequently trigger an intracellular response (Dieter and Marmé, 1988).

The most widely studied calcium binding protein is calmodulin (CaM), a highly conserved, ubiquitous, eukaryotic protein that undergoes a conformational change after binding one to four molecules of calcium (Klee, 1988). This conformational change typically alters the ability of CaM to interact with other proteins thereby modulating their function. For example, $\mathrm{Ca}^{2+}-\mathrm{CaM}$ complexes activate several enzymes in vitro (Anderson and Cormier, 1978; Dieter and Marmé, 1981; Ranjeva et al., 1983) and these biochemical activities are believed to be of regulatory importance in vivo. It has been widely reported that the binding of $\mathrm{Ca}^{2+}$ to CaM produces an activation complex, but it is also known that CaM by itself is capable of stimulating some enzymes (Polya 1983; Roberts et al., 1983; Van Eldik et al., 1983). Additionally, the binding of CaM to chloroplast-membrane fractions (Roberts et al., 1983), as well as to fibroblast proteins (Van Eldik and Burgess, 1983), can occur in a $\mathrm{Ca}^{2+}-$ 
independent manner. Thus, is seems that CaM can function as a positive affector under conditions where $\mathrm{Ca}^{2+}$ concentrations are either high or low.

In addition to altering numerous enzymatic activities in vitro CaM, in the presence of $\mathrm{Ca}^{2+}$, can affect plant cortical MTs in situ (CYr, 1991). This suggests that. $\mathrm{Ca}^{2+}$, via CaM, may integrate MT dynamics in the cortical MT array with other cellular activities. The goal of this study was to increase our understanding about the interactions between calcium, CaM and this array.

Previous work by Cyr (1991) provided in situ data to implicate $\mathrm{Ca}^{2+}$-CaM in the regulation of the cortical MT array. However, while the immunocytochemical work of others failed to link CaM to this array, they did localize it to the spindle apparatus, phragmoplast and perhaps the pre-prophase band (Vantard et al., 1985; Wick et al., 1985). It seemed possible that the inability to demonstrate CaM in the cortical MT array was due to the use of non-optimal antibodies. To address this possibility, we produced a monoclonal antibody to plant CaM for immunocytochemical studies. Immunolocalizations, utilizing protoplasts and suspension cells, have revealed that CaM is associated not only with the arrays described above, but also with the cortical Mt array.

Surprisingly, the absence of calcium strongly influenced the ability to optimally demonstrate cam in the phragmoplast, spindle apparatus and cortical array. The possibility that these results 
indicate the presence of both calcium dependent and independent interactions for CaM on MTs is discussed.

\section{MATERIAL AND METHODS}

Plant Material And Protoplast Preparation

Carrot (Daucus carota $\underline{L}_{\text {.) }}$ ) and tobacco (Nicotiana tobacum I. var. Bright Yellow 2) suspension cells were cultured as previously described (Nagata et al., 1981; Cyr and Palevitz, 1989). Suspension-cultured cells were converted into protoplasts using standard enzymatic methods (Cyr, 1991). Incubation in enzymes did not exceed $3 \mathrm{hr}$. After conversion, protoplasts were filtered through cotton, collected by centrifugation at $300 . \mathrm{g}$ for $5 \mathrm{~min}$, and washed twice in PM buffer $(50 \mathrm{mM}$ Pipes, $\mathrm{pH} 6.9 ; 1 \mathrm{mM}$ $\mathrm{MgSO}_{4} ; 1 \mathrm{mM}$ EGTA) with $0.4 \mathrm{M}$ mannitol added as an osmoticum. PM buffer supplemented with mannitol is referred to as PMM.

In experiments where stabilization of MTs was necessary, taxol was added to $10 \mu \mathrm{M}, 20 \mathrm{~min}$ before enzyme removal. Conversely, for the depolymerization of MTs, amiprophos-methyl (APM) was added to $20 \mu \mathrm{M}, 45 \mathrm{~min}$ before enzyme removal.

\section{Lysing and Fixing protoplasts}

Protoplasts, resuspended in PMM, were settled onto poly-L-lysine coated slides (applied as a $1 \mathrm{mg} / \mathrm{mL}$ solution, 300,000 D; Sigma Chemicals, St. Louis, MO) for $5 \mathrm{~min}$. PMM was removed by capillary action and detergent lysis buffer applied 
for $5 \mathrm{~min}$. Lysis buffer consisted of; $50 \mathrm{mM}$ Pipes ( $\mathrm{pH} 6.9$ ), 1 $\mathrm{mM} \mathrm{MgSO}_{4}, 1 \mathrm{mM}$ EGTA, $10 \mathrm{mM} \mathrm{3-[(3-Cholamidopropyl)-}$ dimethylammonio]-1-propane-sulfonate (CHAPS) detergent, and 10 $\mu \mathrm{g} / \mathrm{mL}$ each of the following protease inhibitors: antipain, aprotinin, chymostatin, pepstatin, and leupeptin. $\mathrm{CaCl}_{2}$ replaced EGTA in experiments involving high levels of calcium (above 100 $\mu M)$. An EGTA/calcium buffer system was employed when the required calcium level was less than $100 \mu \mathrm{M}$. For experiments in which the cells were osmotically lysed, CHAPs and mannitol were omitted.

After lysis, the extracted cells were fixed for $20 \mathrm{~min}$ using: $4 q$ formaldehyde ( $w / v$; made fresh from paraformaldehyde), $0.1 \%$ glutaraldehyde $(\mathrm{v} / \mathrm{v}), 50 \mathrm{mM}$ Pipes $(\mathrm{pH} 6.9), 1 \mathrm{mM} \mathrm{MgSO}_{4}, 5 \mathrm{mM}$ EGTA. $\mathrm{CaCl}_{2}$ replaced EGTA when experiments involved calcium. The fixative was removed by capillary action and the slides containing the lysed, fixed protoplasts were washed 15 min with PBS.

Fixation of Suspension Cultured Cells

Culture media was removed from 5 day-old cell suspension by centrifugation at $300 \cdot \mathrm{g}$ for $5 \mathrm{~min}$. Fixatives identical to those described for protoplasts were applied for $1 \mathrm{hr}$. Cells were rinsed once in PBS, settled onto poly-L-lysine coated slides, then permeabilized as described in Kuss-Wymer and Cyr (1992) with minor modifications. Briefly, the permeabilization solution contained $0.5 q$ Cellulase YC $(w / v), 0.05 q$ Pectolyase (w/v; Seishin Pharmaceutical Co., Ltd, Tokyo) $0.05 \%$ Triton X-100 
$(v / v), 0.25 M$ mannitol in PM buffer and $1 \mu \mathrm{g} / \mathrm{mL}$ each of the protease inhibitors mentioned above. Cells were rinsed for 10 min in PBS containing $0.05 \%$ Tween $20(\mathrm{~V} / \mathrm{V})$ and $0.25 \mathrm{M}$ mannitol.

\section{Immunolocalization of Mts}

After fixation, cells and 1.ysed protoplasts were blocked with $3 \%$ BSA dissolved in PBS $(w / v)$ and incubated in one of three primary antibodies (anti-carrot tubulin, designated mAb1F8; anti-soybean tubulin, Cyr et al., 1987; anti-carrot CaM, designated mAb1D10) for at least $45 \mathrm{~min}$. After rinsing for 15 min in PBS, they were incubated in an appropriate secondary antibody, conjugated with FITC, for at least $45 \mathrm{~min}$, followed by a 15 min rinse with PBS. The slides were mounted in $4 \mathrm{M}$ glycerol, $100 \mathrm{mM}$ Tris ( $\mathrm{pH} 9.0$ ) containing $1 \mathrm{mg} / \mathrm{mL}$ phenylenediamine and $1 \mathrm{mg} / \mathrm{mL}$ Hoescht 33258 (Calbiochem, La Jolla, CA). The slides were viewed with a Zeiss Axioskop (Carl Zeiss, Thornwood, N.Y.) equipped with a $150 \mathrm{~W}$ xenon epifluorescent iliuminator and a 100x objective. Photomicrographs were obtained using Tri-X film, which was exposed and developed nominally.

\section{Production of Monoclonal Antibodies}

CaM was purified to apparent homogeneity from carrot suspension cells using methods described. in Cyr (1991). Mice (Balb/C females, 6 weeks of age) were immunized on day 0 with 100 $\mu \mathrm{g}$ of CaM emulsified in complete Freund's adjuvant (Calbiochem, La Jolla, CA). On days 14 and 28 the animals were challenged 
with $50 \mu \mathrm{g}$ of CaM emulsified in incomplete Freund's adjuvant. On day 40 the animals were test bled and the one showing the highest serum titer was selected and injected intraperitoneally with 25 $\mu \mathrm{g}$ of CaM in $\mathrm{PBS}$ on days 3, 2, and 1 prior to fusion. After euthanasia by cervical dislocation, the spleen was aseptically removed and the splenocytes obtained using standard methods (Zola, 1987) .

Hybridoma cells were produced by fusing splenocytes with a myeloma cell line (ATCC \#CRL 1580, named X63-Ag.653; sometimes referenced as $P 3 A 63$ ) using $P E G$ j.nduced fusion according to standard procedures (Marusich, 1988) and the fusion products screened using HAT selection. Supernatants from wells harboring hybridoma colonies were assayed for CaM antibodies using ELISA.

\section{Electrophoresis And Immunoblotting}

Total proteins were obtained from suspension cultured cells by explosive decompression using a French Press at 5,000 PSI as described in Cyr and Palevitz (1989). Tubulin was obtained by further processing using DEAE ion-exchange chromatography by the method of Morejohn and Fosket (1982) as modified by Cyr and Palevitz (1989). Proteins were electrophoresed by SDS-PAGE using $15 \%(w / v)$ separating gels according to the method of Laemmli (1970). For immunoblot analysis, the proteins were transferred either onto nitrocellulose membranes (Bio-Rad, Richmond, CA) using the method of Towbin et al. (1979), or onto PVDF membranes (Immobilon P; Millipore Corp, Bedford MA) using 
the method of Hulen et al. (1991). After transfer, the membranes were blocked overnight with $38 \mathrm{BSA}$ in $\mathrm{PBS}(w / v)$ and reacted for a minimum of $1 \mathrm{hr}$ at room temperature with the undiluted hybridoma supernatant. The blots were then washed in three changes of PBS +0.058 Tween-20 (v/v) and incubated with a goat anti-mouse IgG conjugated to alkaline phosphatase, diluted 1:1000 in PBS $+3 \%$ $\operatorname{BSA}(w / v)$ for a minimum of $1 \mathrm{hr}$ at room temperature. After washing as before, the membranes were briefly rinsed in substrate buffer $\left(0.1 \mathrm{M}\right.$ Tris, $\left.\mathrm{pH} 9.5,1 \mathrm{mM} \mathrm{MgCl}_{2}\right)$ and incubated at room temperature using the method of Blake et al. (1984).

\section{RESULTS}

CaM is reported to be a poor antigen, presumably because it is small, acidic and very highly conserved (Bulen et al., 1991). Not surprisingly, we found relatively few positive hybridoma colonies that secreted antibodies which could be detected using ELISA. A total of 610 colonies (from two fusions) were screened and 19 of these colonies gave a positive, but often weak response. Table I lists ELISA data for five positive colonies which were cloned by limiting dilution. The clone designated as mAb1D10 gave the best combined signals with ELISA, immunocytochemistry and immunoblotting, and therefore was used in all subsequent experiments. Antibody isotyping protocols revealed that mAb1D10 is an IgG.

Consistent with previous reports (Van Eldik and Wolchok, 1984; Hincke, 1988; Hulen et al., 1991), it was difficult to 
obtain satisfactory CaM signals using standard immunoblot methods (Towbin et al., 1979) due to the poor adherence of CaM to nitrocellulose membranes. However, satisfactory results were obtained utilizing a PVDF membrane and phosphate transfer buffer followed by glutaraldehyde fixation (Hulen et al., 1991). Figure 1 shows that mAb1D10 recognized two closely migrating polypeptides amongst total carrot proteins that comigrated with purified carrot CaM. Similar results were observed using total tobacco proteins (data not shown). Electrophoretic variants of CaM are not unusual (williams et al. 1984). Jablonsky et al., (1991) showed that CaM, from a variety of plant sources, often migrates as a major and minor doublet, with the slow migrating form representing a post-translational modification in which a terminal lysine residue is removed from the carboxy-terminus. Indirect immunofluorescent staining patterns from immunolocalizations with mAb1D10 on lysed carrot protoplasts revealed filamentous signals similar to those seen with antitubulin antibodies (Fig. 2, A and B). A fluorescent signal was not observed when culture media was substituted for the hybridoma supernatant (data not shown). Additionally, similar distribution patterns were found in tobacco protoplasts (Fig. 2, C and D), which are larger than carrot protoplasts and consequently lend themselves better to photodocumentation. Therefore, the figures in this paper depict results obtained from tobacco cells, but in all experiments, carrot cells were examined and found to behave similarly. 
One caveat of visualizing antigens in detergent-lysed cells, is that artifactual rearrangements can occur during lysis (Melan and sluder, 1992). To investigate this possibility, in relation to the filamentous pattern observed with mAb1D10, protoplasts were lysed osmotically (in the absence of detergent and mannitol) prior to fixation and then processed for immunslocalization. Again, a filamentous pattern was observed (data not showa), indicating that the inclusion of detergent did not cause artifactual redistribution of the antigen.

The filamentous pattern observed with mAb1D10 is similar to the MT localization seen with anti-tubulin antibodies. In order to verify that this staining pattern represented the localization of CaM to MTs, cells were incubated with the MT-destabilizing herbicide amiprophos-methyl (APM; Morejohn and Fosket, 1984) prior to lysis. These conditions resulted in the obliteration of MTs and consequently in a loss of fluorescent signal with both mAb1D10 and the anti-tubulin antibodies (Fig. 3, A and B). This data indicates that CaM associates with cortical MTs.

It is important to note that the above observations were made when protoplasts were lysed in the presence of EGTA. CaM typically binds to proteins in a calcium-dependent manner, therefore we wanted to determine what effect calcium would have on the ability to visualize CaM associated with cortical MTs. However, because MTs are sensitive to calcium (Cyr, 1991), it was necessary to stabilize the MTs with the drug taxol prior to the addition of calcium. It is notable that a decrease in cam- 
specific signal was observed at $1 \mu \mathrm{M} \mathrm{Ca}{ }^{2+}$ (Fig. 4A) and at $3.6 \mu \mathrm{M}$ $\mathrm{Ca}^{2+}$ the signal was no longer demonstrable on the taxolstabilized MTs (Fig. 4B;. However, MTs were clearly present as evidenced with the anti-tubulin antibodies (Fig. 4C). Furthermore, higher concentrations of calcium (upwards to $1 \mathrm{mM}$ ) gave negative immunocytochemical results with mAb1D10 (data not shown).

It is possible that the failure to immunolocalize CaM to cortical MTs, in the presence of calcium, is the result of epitope masking that occurs as a result of a calcium-dependent conformational change in CaM (Harper, 1983). To investigate if the CaM epitope recognized by mAb1D10 behaves cryptically in the presence of calcium, the binding characteristics for this antibody were determined in the presence and absence of calcium. The antibody has a somewhat higher affinity for CaM in the presence of calcium in the ELISA (Fig. 5).

Another possible explanation for the inability to immunolocalize CaM in a calcium-dependent fashion, is that a CaM binding protein could associate with CaM and sterically interfere with the binding of mAb1D10. If this hypothesis is correct then it should be possible to conceal the epitope in the presence of calcium and then expose it by chelation with EGTA. Experimentally this was done by lysing taxol-treated protoplasts in the presence of calcium and then rinsing the extracted cells with a large excess of calcium-free buffer (containing EGTA). The preparations were then fixed and processed for 
immunolocalization. These protoplasts did not have significant amounts of CaM in association with the cortical MTs (Fig. 6A), even though MTs were clearly present as evidenced by tubulin immunolocalizations treated identically (Fig. 6B). This indicates that the failure to immunolocalize CaM in the presence of calcium was not due to a blocking event in which a CaM-binding protein obscured the epitope recognized by mAb1D10. Rather, the inability to detect CaM after exposure to $\mathrm{Ca}^{2+}$ is due to its disassociation from MTs.

Protoplasts were chosen as our main experimental system because background noise caused by soluble cytosolic CaM was significantly less than that in intact cells. In addition, the cell wall is capable of sequestering large amounts of calcium (Demarty et al., 1984) therefore, protoplasts provided a cell type in which calcium concentrations could be known with a reasonable degree of certainty, especially when comparing treatments which included EGTA or exogenous calcium. However, suspension cultured cells were also examined. Although it was more difficult to discern and document the association of CaM with cortical MTs in intact suspension cultured cells, mAb1D10 did immunolocalize to these MTs (Fig. 7A), as well as to those in squashed apical meristematic root cells (data not shown). However, as shown with protoplasts, CaM only localized to MTs in the presence of EGTA. When cells were pretreated with $20 \mathrm{mM}$ $\mathrm{CaCl}_{2}$, the antibody no longer reacted with the MTs (Fig. 7B). In these experiments, high external calcium concentrations were used 
because plant cells have efficient $\mathrm{Ca}^{2+}$ pumps which maintain cytoplasmic concentrations at low levels (Hepler and Wayne, 1985). While the addition of such high $\mathrm{Ca}^{2+}$ concentations drives cytoplasmic $\mathrm{Ca}^{2+}$ upwards, the actual magnitude of this increase is uncertain. Immunolocalizations with anti-tubulin antibodies indicated that normal appearing MTs were present in all cells examined.

As previously repo:ted (Vantard et al., 1985; Wick et al., 1985), CaM associates with the mitotic apparatus, however, we found this association was also highly iniluenced by the addition of calcium. In the presence of EGTA, CaM localized to the spindle apparatus with readily discernable kinetechore MTs (Fig. 8A), while in the presence of calcium, the spindle-associated fluorescence was considerably reduced and lacking in MT-detail (Fig. 8C). CaM was also observed in the phragmoplast (data not shown).

A number of drugs affect the function of CaM (Allan and Hepler, 1989). Two drugs, trifluoperazine and W7, were applied to protoplasts to investigate if they altered CaM localization. Protoplasts, treated with $20 \mu \mathrm{M}$ trifluoperazine, had a variable diminished signal in the cortical arrays when compared to untreated controls (data not shown) and therefore their usefullness in future studies is uncertain. No inhibition of microtubule signal attributable to CaM was evidenced with $20 \mu M$ W7 


\section{DISCUSSION}

It is possible that calcium, in conjunction with CaM, aids in orchestrating MT function with other cellular events in higher plants. An earlier report showed that calcium, via CaM, affects the behavior of cortical MTs in lysed carrot protoplasts (Cyr, 1991). The observation that CaM alters the stability of cortical MTs was puzzling because previous reports did not reveal immunolocalizations of CaM to these MTs.

This study has shown, via immumocytochemical techniques, the localization of CaM to the cortical MT array. There are at least two possibilities why other immunocytochemical studies did not detect this association. First, the antibody used in this study may have a relatively high affinity for plant CaM, which permits the detection of lower amounts of antigen. This, combined with improved microscope optics may have enhanced the visualization of this association. Second, the antibodies used in previous studies may have had a low affinity for the calciumfree form of CaM. Either possibility (or both) would have made it difficult to detect CaM in the cortical MT array.

Monoclnnal antibodies recognize a single epitope which can be shared with other proteins. For example, calcium dependent protein kinase (CDPK) shares some homology with CaM and localizes to the microfilament component of the cytoskeleton (Putnam-Evans et al., 1989). However, mAb1D10 staining patterns are dependent upon intact MTs and immunoblot analysis of total proteins revealed two closely migrating polypeptides that comigrated with 
purified plant CaM (CDPK has a molecular mass of about 53,000 D). It is most plausible that the results presented in this study represent an authentic intracellular localization of CaM and not to a protein possessing a similar epitope.

It is remarkable that the ability to localize CaM to cortical MTs is dependent upon the absence of calcium and that the signals associated with the spindle and phragmoplast were also markedly enhanced. One might ask if the failure to efficiently immunolocalize CaM in the presence of calcium is due to a detection artifact rather than reflective of the in vivo situation. It should be noted that mabldio showed a slightly higher affinity to CaM in the presence of calcium with the ELISA, indicating that if any detection bias exists, it is to under estimate the amount of CaM bound in the absence of calcium.

The data presented in this manuscript indicates that at low calcium concentrations, CaM associates strongly with MTs however, when calcium concentrations increase, its affinity for MTs decreases and CaM disassociates more readily from MTs. The concentration at which CaM begins to lose its affinity for MTs in vivo is unknown however, CaM associated with cortical MTs was difficult to detect in most protoplasts at $1 \mu \mathrm{M}$ calcium. At 3.6 $\mu M$ calcium, it was impossible to find protoplasts which showed this association. Further studies (perhaps using microinjected fluorescently derivatized CaM) are required to determine the in vivo association characteristics of CaM with MTs. We predict that at "average" or "resting" intracellular calcium 
concentrations $\left(10^{-7} \mathrm{M}\right)$, CaM associates with MTs and as calcium concentrations increase $\left(\geq 10^{-6}\right)$ CaM disassociates. Regardless of the precise affinities that CaM has for MTs, it is perhaps more important to consider the relevance of the association. Cyr (1991) demonstrated that protoplasts, lysed in the presence of calcium, have unstable MTs. However, protoplasts lysed in the presence of EGTA, possess MTs which are insensitive to subsequent calcium exposure; further, the calcium-sensivity of these MTs can be reconstituted with the addition of $\mathrm{Ca}^{2+}$ and $\mathrm{CaM}$. Hence, this earlier work indicates the presence of $\mathrm{Ca}^{2+}$-dependant $\mathrm{CaM} / \mathrm{MT}$ interaction.

The present immunocytochemical data showing calciumindependent binding of CaM to MTs, together with the previous reconstitution data indicating $\mathrm{Ca}^{2+}$-dependent $\mathrm{CaM} / \mathrm{MT}$ interactions, suggests that MTs possess two binding sites for CaM: one calcium dependent, and the other independent. This hypothesis has an underlying corollary, namely, MTs that have CaM bound in a calcium-free state are stable, whilst those binding CaM in a calcium-dependent state are unstable. Although the data is consistant with the existance of two types of Cam/MT interactions, the molecular character of these two putative binding sites is unknown.

Reports indicate that CaM can bind directly to tubulin (Kumagai et al., 1979). However, because of the relative low affinity of this interaction, it is unclear whether this association occurs in vivo. It is more likely that 
physiologically relevant interactions occur via interactions with MT-binding proteins. Such interactions can be demonstrated biochemically and the best characterized interaction of this type is between CaM and a protein referred to as stable Tubule only Protein (STOP). Interestingly, in the presence of $\mathrm{Ca}^{2+}-\mathrm{CaM}$, STOPs disassociate from MTs, and concomitantly, the tubulin polymer loses its cold-stable characteristic (Margolis et al, 1986). STOPs have not been found in plants, but homologs may exist.

MT-binding proteins, which bind CaM in a calcium-dependent fashion, have been identified (Cyr, 1991) therefore, future experiments should concentrate on identifying binding proteins to which CaM binds in a calcium-independent fashion as well. In vitro MT assembly assays should then reveal if these two classes of proteins have opposing activities upon MT-assembly and/or dynamics. 
LITERATURE CITED

Alan E, Hepler, PK (1989) Calmodulin and calcium-binding proteins. In PK Stumpf, ed, The Biochemistry of Plants. Academic Press, New York, pp 455-484

Anderson JM, Cormier MJ (1978) Calcium-dependent regulator of NAD kinase in higher plants. Biochem Biophys Res Commun 84: $595-602$

Blake MS, Johnston KH, Russel-Jones GJ, Gotschlich EC (1984) A rapid, sensitive method for detection of alkaline phosphatase-conjugated anti-antibody on Western blots. Anal Biochem 136: 175-179

Cyr RJ (1991). Calcium/calmodulin affects microtubule stability in lysed protoplasts. J Cell Sci 100: 311-317

Cyr RJ, Bustos MM, Guiltinan MJ, Fosket DE (1987) Developmental modulation of tubulin proteins and mRNA levels during somatic embryogenesis in cultured carrot cells. Planta $171: 365-376$

Cyr RJ, Palevitz BA (1989) Microtubule-binding proteins from carrot. I. Initial characterization and microtubule bundling. Planta 177: 245-260

DeMarty M, Morvan C, Thellier M (1984) Calcium and the cell wall. Plant Cell Environ 7: 441-448

Dieter P, Marmé D (1988) The history of calcium-binding proteins. In MP Thompson, ed, Calcium-binding Proteins: Volume 1, Characterization and Properties. CRC Press, Boca Raton, FL, pp 1-9 
Dieter P, Marmé D (1981) A calmodulin-dependent, microsomal ATPase from corn (Zea mays L.). FEBS Lettr 125: 245-248 Gilroy S, Read ND, Trewavas AJ (1990) Elevation of cytoplasmic calcium by caged calcium or caged inositol triphosphate initiates stomatal closure. Nature 346: 769-771

Harper JF (1983) Antigenic structure of calmodulin: production and characterization of antisera specific for plant calmodulins or $\mathrm{Ca}^{2+}$-replete vs. $\mathrm{Ca}^{2+}$-free calmodulins. J Cyclic Nucleo Prot Phos Res 9: 3-17

Hepler PK, Wayne RO (1985) Calcium and plant development. Ann Rev Plant Physiol 36: 397-439

Hincke MT (1988) Conditions for improved adsorption of calmodulin to nitrocellulose: Detection by ${ }^{45} \mathrm{Ca}$ binding. Electrophoresis 9: 303-306

Hulen D, Baron A, Salisbury J, Clarke M (1991) Production and specificity of monoclonal antibodies against calmodulin from Dictyostelium discoideum. Cell Motility and Cytoskel 18: $113-122$

Jablonsky PP, Grolig F, Perkin JL, Williamson RE (1991) Properties of monoclonal antibodies to plant calmodulin. Plant Sci $76: 175-184$

Klee $\mathrm{CB}$ (1988) Interaction of calmodulin with $\mathrm{Ca}^{2+}$ and target proteins. In $\mathrm{P}$ Cohen, $C B$ Klee, eds, Calmodulin: Volume 5, Molecular Aspects of Cellular Regulation. Elsevier, Amsterdam, pp 35-56

Kumagai H, Nishida E (1979) The interactions between calcium- 
dependent regulator protein of cyclic nucleotide phosphodiesterase and microcubule proteins. II. Association of calcium-dependent regulator protein with tubulin dimer. $\mathrm{J}$ Biochem 85: 1267-1274

Kuss-Wymer C, Cyr R (1992) Tobacco protoplasts differentiate into elongate cells without net microtubule depolymerization. Protoplasma 168: 64-72

Laemmli OK (1970) Cleavage of structural protein during the assembly of the head of bacteriophage T4. Nature 227: 680685

Margolis RL, Rauch CT, Job D (1986) Purification and assay of a $145 \mathrm{kDs}$ protein $\left(\operatorname{STOP}_{145}\right)$ with microtubule-stabilizing and motility behavior. Proc Natl Acad Sci USA 83:639-643

Marmé D (1988) The role of calcium in the regulation of plant cellular metabolism. In Ch. Gerday, L Bolis, R Gilles, eds, Calcium and Calcium Binding Proteins: Molecular and Functional Aspects. Springer-Verlag;, Berlin Heidelberg, pp 201-208

Marusich MR (1988) Efficient hybridoma production using previously frozen splenocytes. J Immunol Meth 114: 155-159 Melan MA, Sluder G (1992) Redistribution and differential extraction of soluble proteins in permeabilized cultured cells. Implications for immunofluoresence microscopy. J Cell Sci 101: 731-743 
Morejohn LC, Fosket DE (1982) Higher plant tubulin identified by self-assembly into microtubules in vitro. Nature 297: 426428

Morejohn LC, Fosket DE (1984) Inhibition of plant microtubule polymerization in vitro by the phosphoric amide herbicide amiprophos-methyl. Science 224: 874-876

Nagata T, Okada K, Takebe I, Matsui C (1981) Delivery of tobacco mosaic virus RNA into plant protoplasts mediated by reversephase evaporation vesicles (liposomes). Mol Gen Genet 184: $161-165$

Putnam-Evans C, Harmon AC, Palevitz BA, Fechheimer M, Cormier MJ (1989) Calcium-dependent protein kinase is localized with Factin in plant cells. Cell Motil Cytoskel 12: 12-22

Polya GM (1983) Calmodulin stimulates a $\mathrm{Ca}^{2+}$-independent plant protein kinase. Biochem Int $7: 339-344$

Ranjeva R, Refeno G, Boudet AM, Marmé D (1983) Activation of plant quinate: $\mathrm{NAD}^{+}$3-oxidoreductase by $\mathrm{Ca}^{2+}$ and calmodulin. Proc Natl Acad Sci USA 80: 5222-5224

Roberts DM, Zielinski RE, Schleicher M, Watterson DM (1983) Analysis of suborganeller fractions from spinach and pea chloroplasts for calmodulin-binding proteins. J Cell Biol 97: $1644-1647$

Towbin H, Staehelin I, Gordon J (1979) Electrophoretic transfer of proteins from polyacrylamide gels to nitrocellulose sheets: Procedure and some applications. Proc Natl Acad Sci USA $76: 4350-4354$ 
Van Eldik LJ, Burgess $\mathrm{WH}$ (1983) Analytical subcellular distribution of calmodulin and calmodulin-binding proteins in normal and virus-transformed fibroblasts. J Biol Chem 258: $4539-4545$

Van Eldik LJ, Wolchok SR (1984) Conditions for reproducible detection of calmodulin and $5100 \beta$ in immunoblots. Biochem Biophys Res Commun 124: 752-759

Vantard M, Lambert A-M, De Mey J, Pioquot P, Van Eldik LJ (1985) Characterization and immunocytochemical distribution of calmodulin in higher plant endosperm cells: Localization in the mitotic apparatus. J Cell Biol 101: 488-499

Wick SM, Muto S, Duniec J (1985) Double immunofluorescence labeling of calmodulin and tubulin in dividing plant cells. Protoplasma 126: 198-206

Williams DC, O'Riordan, Beattie RE, O'Neil C (1984) Multiple forms of calmodulin on sodium dodecyl sulphate/polyacrylamide-gel electrophoresis. Biochem Soc Trans 12: $472-474$

Zola H (1987) Making hybridomas. In H Zola ed, Monoclonal Antibodies: A Manual of Techniques. CRC Press, Boca Raton, FL, pp 39-44 
LEGENDS TO FIGURES

Figure 1. The antibody, mab1D10, is specific for calmodulin. One microgram of carrot CaM (lane 1) and $50 \mu \mathrm{g}$ of total carrot proteins (lane 2) were separated by SDS-PAGE using a $15 \% \mathrm{gel}$. The polypeptides were transferred to PVDF membranes and immunoblotted using mAb1D10. The migrated positions of reference polypeptides are indicated on the right and correspond to $M_{r}$ values of: 110,$000 ; 84,000 ; 47,000 ; 20,100 ; 14,200$.

Figure 2. Immunolocalization patterns using mAb1D10 on lysed protoplasts are similar to that seen with anti-tubulin antibody. Carrot (panels A \& B) or tobacco (panels C\& D) protoplasts were reacted with either mAb1D10 (panels $A \& C$ ) or an anti-tubulin antibody (panels B \& C). These protoplasts were isotonicallylysed with detergent in the presence of $1 \mathrm{mM}$ EGTA. Bar $=10 \mu \mathrm{m}$.

Figure 3. The filamentous staining pattern observed with mAb1D10 is dependent upon intact MTs. Tobacco protoplasts were treated with the anti-microtubule drug APM, isotonically-lysed in the presence of detergent, and reacted with either mAb1D10 (panel A) or an anti-tubulin antibody (panel B). Bar $=10 \mu \mathrm{m}$.

Figure 4. Calmodulin binds to cortical MTs in a calciumindependent manner. Taxol-treated tobacco protoplasts were detergent-lysed in the presence of $1 \mu \mathrm{M} \mathrm{Ca}{ }^{2+}$ (panel A), $3.6 \mu \mathrm{M}$ $\mathrm{Cl}^{2+}$ (panel B), or $500 \mu \mathrm{M} \mathrm{Ca}{ }^{2+}$ (panel C) and immunolocalized with 
mAb1D10 (panels $A \& E$ ) or an anti-tubulin antibody (panel C). Bar $=10 \mu \mathrm{m}$.

Figure 5. The monoclonal antibody, 1D10, binds calmodulin in the presence and absence of calcium. Different amounts of carrot calmodulin were adsorbed overnight in microtiter plates in the presence of $1 \mathrm{mM}$ calcium (closed circles) or in its absence (1 $\mathrm{mM}$ EGTA; open triangles). The plates were processed for ELISA as in Fig. 1, except calcium or EGTA was maintained in all solutions and Tris-buffered saline substituted for PBS. Each data point represents the mean value from three triplicate assays. Negative resu?t:s were observed with bovine calmoaulin. Stardard deviation bars are not shown because they are smaller than the symbols.

Figure 6. Calmodulin disassociates from MTs in the presence of calcium. Taxol-stabilized, tobacco protoplasts were detergertlysed in the presence of $500 \mu \mathrm{M}$ calcium, then rinsed and fixed with EGTA-containing buffers. The lysed protoplasts were immunolocalized with mAb1D10 (panel A) or anti-tubulin antibndy $($ parel B). Bar $=10 \mu \mathrm{M}$.

Figure 7. The association of calmodulin to MTs is observed in intact suspension cultured cells. Cultured tobacco cells were pretreated without (panel A) or with (panel B) $20 \mathrm{mM}$ calcium, then fixed and processed for immunolocalization with mab1D10. Bar $=10 \mu \mathrm{m}$. 
Figure 8. Calcium affects the amount of calmodulin that associates with spindle MTs. Taxol-stabilized protoplasts were detergent-lysed in the absence of $\mathrm{Ca}^{2+}$ (panels $\mathrm{A} \& \mathrm{~B}$ ) or in its presence (500 $\mu$; panels C \& D). After fixation they were immunolocalized with mAb1D10 and stained with the chromatin binding dye, Hoescht 33258. The localization of mAb1D10 is shown in panels $A \& C$, while the chromatin pattern in the identical cells is shown in panels $B \& D$. Bar $=10 \mu \mathrm{m}$. 
Table I. ELISA readings for positive hybridoma colonies One hundred nanograms of carrot CaM, or 100 ug of BSA were absorbed to microtiter plates overnight. After blocking with 0.18 BSA $+0.05 \%$ Tween 20, the hybridoma superuatants were adsorbed for $1 \mathrm{hr}$, washed, and a goat anti-mouse alkaline phosphatase reporter antibody applied for $1 \mathrm{hr}$. The plates were washed and exposed to the colorimetric substrate, p-nitrophenol phosphate at pH 9.5. The absorbance of each well was determined with a microtiter plate reader at $\mathrm{OD}_{405}$.

\begin{tabular}{lll}
\hline Cloned Colony & CaM OD 405 & BSA OD $_{405}$ \\
\hline $1 \mathrm{E} 7$ & 1.137 & 0.009 \\
$5 \mathrm{D} 12$ & 0.223 & 0.006 \\
$4 \mathrm{H} 6$ & 0.118 & 0.009 \\
$10 \mathrm{E} 2$ & 2.608 & 0.002 \\
$1 \mathrm{D} 10$ & 1.479 & 0.010 \\
\hline
\end{tabular}


Pennsylvania State University University Park, PA 16802

Role of $\mathrm{Ca}^{++} / \mathrm{calmodul}$ in in the regulation of microtubules in higher plants

R. Cyr, Department of Biology

In FY92 we continued to define the role of $\mathrm{Ca}^{++} / \mathrm{calmodul}$ in in regulating cortical microtubules (Mts) in higher plants. Enclosed is a manuscript that has recently been submitted to Plant Physiology which describes our immunocytochemical localization of calmodulin to MTs. This work is significant because it demonstrates that calmodulin can bind Mts in a calcium independent fashion. This, coupled to our earlier work (J. Cell Sci. 100:311, 1991) indicates calmodulin can interact in both a calcium dependent, as well as independent fashion with cortical Mts. These two findings are exciting because they raise the possibility that calmodulin may have differing effects upon MTs depending upon the concentration of calcium. Previous to this finding we had focused upon calcium dependent effects of calmodulin upon MTs, however in the future calcium independent effects will also need to be considered. Progress has also been made in three other areas in the last year.

A major thrust of the grant is to define the effects of calcium upon microtubules in vivo. In our initial report, using lysed protoplasts (which is closer to an in vitro observation), we noted Mts to be destabilized by calcium/calmodulin. As we had discussed, this effect could be the result of gross 
depolymerization induced by $\mathrm{Ca}^{++} /$calmodulin, or by an increase in the dynamic flux rate. Joe Sliman, an undergraduate researcher working in the lab is now able to estimate intracellular calcium levels in cultured cells. He has confirmed that elevation of extracellular calcium levels results in an increase in intracellular calcium levels in carrot protoplasts. Debra Fisher, a research assistant in the lab, has found that protoplast possessing elevated intracellular levels of calcium contain highly fragmented cortical Mts. Interestingly, the mitotic and phragmoplast Mts are relatively unaffected. These results indicate that calcium, in conjunction with calmodulin functions to destabilize sensitive microtubules. Further experiments are planned in the upcoming year to further document this work not only in protoplasts, but also in intact cells of both carrot and tobacco.

The second area of interest is to identify the Mt proteins that are affected by $\mathrm{Ca}^{++} /$calmodulin. With this goal in mind Neil Durso, a graduate student in the lab, has isolated Mtbinding proteins using tubulin affinity chromatography. These proteins were then adsorbed to a second column containing calmodulin covalently bound to the matrix. Using this technique, proteins can be identified that absorb in either a calcium dependent, or independent fashion (see attached figure). The MT/calmolulin binding proteins were then assayed for their ability to affect Mt function using in vitro assays. The calcium dependent calmodulin-binding proteins contained a great deal of Mt-bundling activity. This activity was sensitive to the 
presence of $\mathrm{Ca}^{++} / \mathrm{calmodulin}$ which was found to reversibly abolish the bundling activity. This finding is relevant because cortical microtubules (which we have shown to be calcium sensitive) are bundled. It will be important to know at the molecular level how $\mathrm{Ca}^{++} / \mathrm{calmodulin}$ regulates $\mathrm{MT}$-bundling. In the upcoming year we hope to learn more about how this complex interacts with MTbundling proteins.

The third area of pursuit entails defining the proteins involved in mediating the attachment of microtubules to the plasma membrane. Our initial attempts at working with these proteins were hampered by the inability to procure sufficient quantities of proteins with which to work. In the last year alternative methodologies have been developed to obtain greater yields. Attached is a figure which summarizes our current approach. In the upcoming year we will work towards the production of antibodies as well as to define the effect $\mathrm{Ca}^{++} /$calmodulin has on the ability of these proteins to interact with Mts.

In this second year we have continued to make headway into understanding how $\mathrm{Ca}^{++} / \mathrm{calmodul}$ in affects plant microtubules and we are confident that in the upcoming year we will continue to further define the role that $\mathrm{Ca}^{++} / \mathrm{calmodulin}$ plays in mediating microtubule organization in plant cells. 


\section{Tubulin/CaM binding proteins}

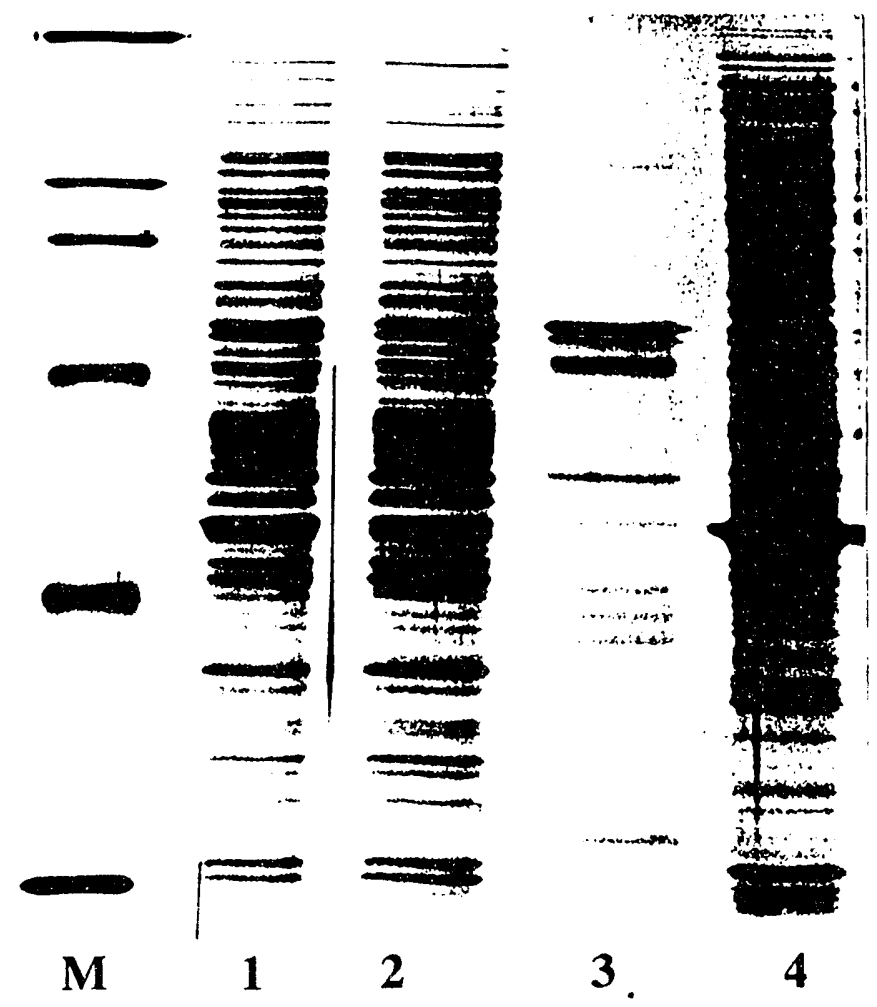

1-Mt-binding proteins before CaM column

2. Unbound proteins

3. Calcium-independent CaM binding proteins

4. Calcium-dependent CaM binding proteins

Soluble carrot proteins were absorbed to a column in which tubulin was covelently bound. The adsorbed proteins were eluted with $0.3 \mathrm{M} \mathrm{NaCl}$ (lane 1). These proteins also bind to taxol stabilized Mts. After dialysis in the absence of calcium the protcins were allowed to absorb to a second column in which calmodulin was covalently bound. Adsorbed proteins were eluted with $1 \mathrm{mM} \mathrm{Ca}^{++}$(lane 3 ). The proteins that did not absorb were reapplied to the column in the presence of calcium. Bound proteins were then eluted with $1 \mathrm{mM}$ EGTA (lane 4). 


\section{Microsomal/MT-binding proteins}

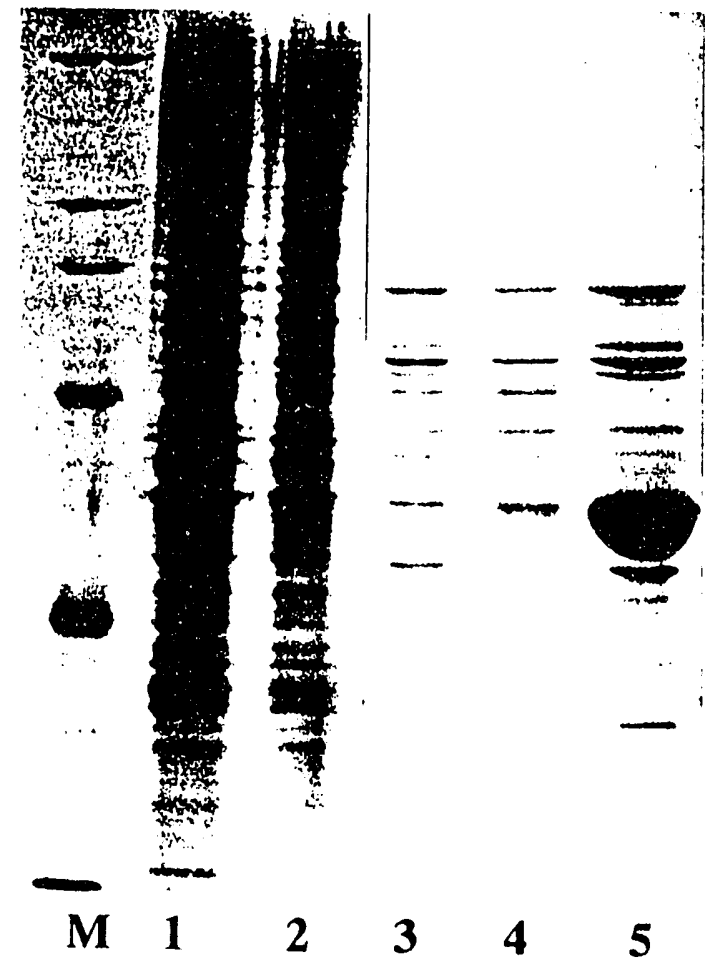

1-Microsome fraction

2-unbound fraction

3-0.3M eluate

4-Supernatant after MT-binding

5-MT pellet

A crude microsomal fraction was prepared from BY-2 tobacco suspension cultured cells. The proteins were solubilized with $10 \mathrm{mM}$ CHAPs detergent (lane 1) and absorbed onto a column to which tubulin was covalently bound. The absorbed proteins were eluted with $0.3 \mathrm{M} \mathrm{NaCl}$ (lane 3) and dialyzed. The eluted and dialyzed proteins were absorbed to taxol stabilized MTs and sedimented through a sucrose cushion. The supernatant (lane 4) and the pellet (lane 5) were collected and analyzed using SDS-PAGE. 
Pennsylvania state University

University Park, PA 16802

Role of $\mathrm{Ca}^{++} /$calmodulin in the regulation of microtubules in higher plants

R. Cyr, Department of Biology

The cytoskeleton, and in particular its microtubule (Mt) component, participates in several processes that directly affect growth and development in higher plants. Normal cytoskeletal function requires the precise and orderly arrangement of Mts into several cell cycle and developmentally specific arrays. One of these, the cortical array, is notable for its role in somehow directing the deposition of cellulose, the most prominent polymer in the biosphere. Unfortunately, little molecular information is available regarding the formation of these arrays, or the cellular signals to which they respond. It is therefore important to acquire information regarding the molecules which regulate Mts within the different arrays. Experimental data has been obtained to suggest that plant cells use calcium, in the form of a $\mathrm{Ca}^{++} / \mathrm{calmodulin}$ complex, to affect the dynamics of Mts within the cortical array. Owing to the importance of $\mathrm{Ca}^{++}$as a regulatory ion in higher plants we are probing for a putative $\mathrm{Ca}^{++} / \mathrm{Mt}$ transduction pathway which may serve to integrate $\mathrm{Mt}$ activities within the growing and developing plant cell. To aid 
in our investigations we are using a lysed cell model in conjunction with immunocytochemical and biochemical methodologies to dissect how $\mathrm{Ca}^{++} /$calmodulin interacts with Mts to affect their function. The information gained in these studies will be useful in understanding how developmentally important signals are transduced into morphogenic events during plant growth and development. 

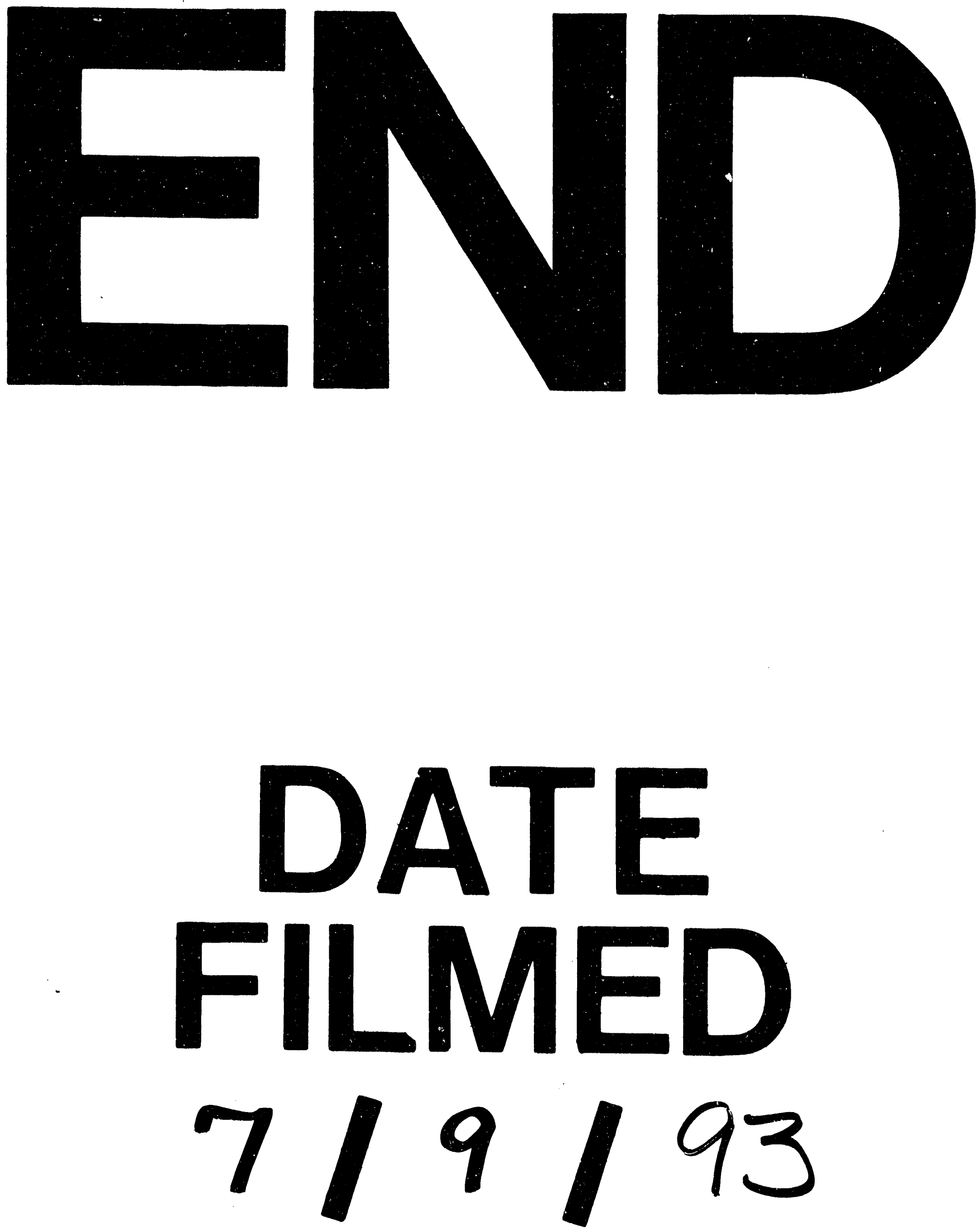
